Available online at http://jnfa.mathres.org
MATHRS Sournal of Nonlinear Functional Analysis
A

\title{
THREE SOLUTIONS FOR A NONLINEAR EQUATION INVOLVING $p$-TRIHARMONIC OPERATORS
}

\begin{abstract}
SAEID SHOKOOH
Department of Mathematics, Faculty of Basic Sciences, Gonbad Kavous University, Gonbad Kavous, Iran

Abstract. The existence of at least three weak solutions for a nonlinear elliptic Navier boundary value problem involving the $p$-triharmonic operator is investigated. The main tools used for obtaining our results are two critical points theorems established in [B. Ricceri, A three critical points theorem revisited, Nonlinear Anal. 9 (2009), 3084-3089] and [G. Bonanno, S.A. Marano, On the structure of the critical set of non-differentiable functionals with a weak compactness condition, Appl. Anal. 89 (2010), 1-10].
\end{abstract}

Keywords. Nonlinear problems; Variational methods; $p$-triharmonic operators.

\section{INTRODUCTION}

The study of the differential equations with $p(x)$-growth conditions is an interesting and attractive topic and has been the objective of considerable attention in recent years; see, e.g., $[1,2,3,4]$ and the references therein. The interest in studying such problems was stimulated by their applications in elastic mechanics, fluid dynamics, the thermo-convective flows of non-Newtonian fluids and the image processing. For more information on modeling physical phenomena by these equations, we refer to $[5,6,7]$.

The operator $\Delta_{p(x)}^{3} u:=\operatorname{div}\left(\Delta\left(|\nabla \Delta u|^{p(x)-2} \nabla \Delta u\right)\right)$ is the $p(x)$-triharmonic operator, where $p(\cdot) \in C(\bar{\Omega})$, which is a natural generalization of the $p$-triharmonic operator (where $p>1$ is a constant).

The following nonlinear Navier boundary value problem involving the $p(x)$-Kirchhoff type triharmonic operator

$$
\left\{\begin{array}{l}
-M\left(\int_{\Omega} \frac{1}{p(x)}|\nabla \Delta u|^{p(x)} d x\right) \Delta_{p(x)}^{3} u=\lambda \zeta(x)|u|^{\alpha(x)-2} u-\lambda \xi(x)|u|^{\beta(x)-2} u, \quad x \in \Omega \\
u=\Delta u=\Delta^{2} u=0, \quad x \in \partial \Omega
\end{array}\right.
$$

E-mail address: shokooh@gonbad.ac.ir.

Received December 22, 2020; Accepted March 10, 2021.

(C)2021 Journal of Nonlinear Functional Analysis 
where $\Omega \subset \mathbb{R}^{N}(N>3)$ is a bounded domain with smooth boundary, $\lambda$ is a positive parameter, $p \in C^{0}(\bar{\Omega})$ with $1<p(x)<\frac{N}{3}$ for any $x \in \bar{\Omega}$ and $\zeta, \xi, \alpha, \beta \in C^{0}(\bar{\Omega})$, was analysed by Rahal in [8].

Motivated by the results, in this paper, we establish the existence of three weak solutions for the following Navier boundary value problem involving the $p$-triharmonic operator:

$$
\left\{\begin{array}{l}
-\Delta_{p}^{3} u=\lambda f(x, u)+\mu g(x, u), \quad x \in \Omega, \\
u=\Delta u=\Delta^{2} u=0, \quad x \in \partial \Omega,
\end{array}\right.
$$

where $\Omega \subset \mathbb{R}^{N}(N>3)$ is a bounded domain with boundary of class $C^{1}, \lambda$ is a positive parameter, $\mu$ is a non-negative parameter, $f, g \in C^{0}(\bar{\Omega} \times \mathbb{R})$ and $p>\max \left\{1, \frac{N}{3}\right\}$.

In recent years, many authors investigated the problem of finding solutions of problems with Navier boundary value condition, we refer the reader to $[1,2,3,4,8,9,10,11,12,13]$. As we see, most of them include $p$ or $p(x)$-biharmonic operators.

In [12], Yin and Liu studied the following $p(x)$-biharmonic elliptic problem with Navier boundary conditions:

$$
\left\{\begin{array}{l}
\Delta_{p(x)}^{2} u=\lambda a(x) f(x, u)+\mu g(x, u), \quad x \in \Omega, \\
u=\Delta u=0, \quad x \in \partial \Omega,
\end{array}\right.
$$

where $\Omega \subset \mathbb{R}^{N}(N \geq 2)$ is a bounded domain with boundary of class $C^{1}, \lambda, \mu$ are non-negative parameters, $p(\cdot) \in C^{0}(\bar{\Omega})$ with $\max \left\{2, \frac{N}{2}\right\}<p^{-}:=\inf _{x \in \bar{\Omega}} p(x) \leq p^{+}:=\sup _{x \in \bar{\Omega}} p(x)$. By the three critical points theorem obtained by Ricceri [14], they established the existence of three weak solutions to problem (1.2).

In [1], by using the critical point theory, the existence of infinitely many weak solutions for a class of Navier boundary value problem depending on two parameters and involving the $p(x)$-biharmonic operator

$$
\left\{\begin{array}{l}
\Delta_{p(x)}^{2} u=\lambda f(x, u)+\mu g(x, u), \quad x \in \Omega, \\
u=\Delta u=0, \quad x \in \partial \Omega,
\end{array}\right.
$$

where $\lambda$ is a positive parameter, $\mu$ is a non-negative parameter, $f, g \in C^{0}(\bar{\Omega} \times \mathbb{R})$ and $p(\cdot) \in$ $C^{0}(\bar{\Omega})$, was discussed.

In [9], Candito and Molica Bisci proved the existence of three weak solutions for the following elliptic Navier boundary problem

$$
\left\{\begin{array}{l}
\Delta\left(|\Delta u|^{p-2} \Delta u\right)=\lambda f(x, u), \quad x \in \Omega, \\
u=\Delta u=0, \quad x \in \partial \Omega,
\end{array}\right.
$$

where $\lambda$ is a positive parameter, $p$ is a constant and $f$ is a suitable continuous function defined on $\bar{\Omega} \times \mathbb{R}$.

To the best of our knowledge, there are just a few contributions to the study of the problems involving $p$-triharmonic operators. The paper is organised as follows. Section 2 , is devoted to our abstract framework. Section 3, the last section, is dedicated to the main results.

\section{PRELIMINARIES}

From now on, we assume that $\Omega$ is an open bounded subset of $\mathbb{R}^{N}, p>\max \left\{1, \frac{N}{3}\right\}$, while

$$
X:=W^{3, p}(\Omega) \cap W_{0}^{1, p}(\Omega)
$$


endowed with the norm

$$
\|u\|=\left(\int_{\Omega}|\nabla \Delta u|^{p} d x\right)^{\frac{1}{p}}
$$

for $u \in X$.

Proposition 2.1 (see [15]). If $\Omega \subset \mathbb{R}^{N}$ is a bounded domain, then the embedding $X \hookrightarrow C^{0}(\bar{\Omega})$ is compact whenever $p>\frac{N}{3}$.

From Proposition 2.1, we see that there exists a positive constant $k$ depending on $p, N$ and $\Omega$ such that

$$
\|u\|_{\infty}=\sup _{x \in \bar{\Omega}}|u(x)| \leq k\|u\|, \quad \forall u \in X .
$$

Corresponding to $f$ and $g$, we introduce the functions $F, G: \Omega \times \mathbb{R} \rightarrow \mathbb{R}$, respectively, as follows

$$
F(x, t):=\int_{0}^{t} f(x, \xi) d \xi, \quad G(x, t):=\int_{0}^{t} g(x, \xi) d \xi
$$

for all $x \in \Omega$ and $t \in \mathbb{R}$.

For every $u \in X$, let us define $\Phi, \Psi, J: X \rightarrow \mathbb{R}$ by putting

$$
\Phi(u):=\frac{\|u\|^{p}}{p}, \quad \Psi(u)=-\int_{\Omega} F(x, u(x)) d x, \quad J(u)=-\int_{\Omega} G(x, u(x)) d x .
$$

By standard arguments, we have that $\Phi$ is Gâteaux differentiable and sequentially weakly lower semicontinuous and its Gâteaux derivative is the functional $\Phi^{\prime}(u) \in X^{*}$, given by

$$
\Phi^{\prime}(u)(v)=\int_{\Omega}|\nabla \Delta u|^{p-2} \nabla \Delta u \cdot \nabla \Delta v d x
$$

for any $v \in X$. Furthermore, the differential $\Phi^{\prime}: X \rightarrow X^{*}$ admits a continuous inverse (see [12, Lemma 3.1]). Similar arguments as those used in [16] imply that $\Psi, J \in C^{1}(X, \mathbb{R})$ with the derivatives given by

$$
\begin{aligned}
& \Psi^{\prime}(u)(v)=-\int_{\Omega} f(x, u(x)) v(x) d x \\
& J^{\prime}(u)(v)=-\int_{\Omega} g(x, u(x)) v(x) d x
\end{aligned}
$$

for any $u, v \in X$. Moreover, thanks to the compact embedding $X \hookrightarrow C^{0}(\bar{\Omega})$, the operator $\Psi^{\prime}$ : $X \rightarrow X^{*}$ is compact.

Our analysis is based on the following theorems. These tools have been successfully applied to different problems in $[2,4,9,13,17]$.

Theorem 2.2 (Ricceri, [14]). Let $X$ be a reflexive real Banach space, and $I \subseteq \mathbb{R}$ an interval. Let $\Phi: X \rightarrow \mathbb{R}$ be a sequentially weakly lower semicontinuous $C^{1}$ functional, bounded on each bounded subset of $X$, whose derivative admits a continuous inverse on $X^{*}$, and let $\Psi: X \rightarrow \mathbb{R}$ be a $C^{1}$ functional with compact derivative. Assume that

$$
\lim _{\|x\| \rightarrow+\infty}(\Phi(x)+\lambda \Psi(x))=+\infty
$$

for all $\lambda \in I$, and there exists $h \in \mathbb{R}$ such that

$$
\sup _{\lambda \in I} \inf _{x \in X}(\Phi(x)+\lambda(\Psi(x)+h))<\inf _{x \in X} \sup _{\lambda \in I}(\Phi(x)+\lambda(\Psi(x)+h)) .
$$


Then, there exist a non-empty open set interval $A \subseteq I$ and a positive real number $q$ with the following property: for every $\lambda \in A$ and every $C^{1}$ functional $J: X \rightarrow \mathbb{R}$ with a compact derivative, there exists $\delta>0$ such that, for each $\mu \in[0, \delta]$, the equation

$$
\Phi^{\prime}(u)+\lambda \Psi^{\prime}(u)+\mu J^{\prime}(u)=0
$$

has at least three solutions in $X$ whose norms are less than $q$.

In the proof of our first main result, we also use the following result to verify the minimax inequality in theorem 2.2.

Theorem 2.3 (Bonanno, [18]). Let $X$ be a non-empty set and $\Phi, \Psi$ two real functions on $X$. Assume that $\Phi(x) \geq 0$ for every $x \in X$ and there exists $u_{0} \in X$ such that $\Phi\left(u_{0}\right)=\Psi\left(u_{0}\right)=0$. Further, assume that exist $u_{1} \in X, r>0$ such that

$\left(\kappa_{1}\right) \Phi\left(u_{1}\right)>r$

$\left(\kappa_{2}\right) \sup _{\Phi(x)<r}(-\Psi(x))<r \frac{-\Psi\left(u_{1}\right)}{\Phi\left(u_{1}\right)}$.

Then, for every $v>1$ and for every $h \in \mathbb{R}$ satisfying

$$
\sup _{\Phi(x)<r}(-\Psi(x))+\frac{r \frac{-\Psi\left(u_{1}\right)}{\Phi\left(u_{1}\right)}-\sup _{\Phi(x)<r}(-\Psi(x))}{v}<h<r \frac{-\Psi\left(u_{1}\right)}{\Phi\left(u_{1}\right)},
$$

one has

$$
\sup _{\lambda \in \mathbb{R}} \inf _{x \in X}(\Phi(x)+\lambda(\Psi(x)+h))<\inf _{x \in X} \sup _{\lambda \in[0, \sigma]}(\Phi(x)+\lambda(\Psi(x)+h))
$$

where

$$
\sigma=\frac{v r}{r \frac{-\Psi\left(u_{1}\right)}{\Phi\left(u_{1}\right)}-\sup _{\Phi(x)<r}(-\Psi(x))} .
$$

Finally, we recall the following tool, obtained by Bonanno and Marano in [19], that we recall in a convenient form.

Theorem 2.4 ([19, Theorem 2.6]). Let $X$ be a reflexive real Banach space. Let $\Phi: X \rightarrow \mathbb{R}$ be a sequentially weakly lower semicontinuous, coercive and continuously Gâteaux differentiable functional whose Gâteaux derivative admits a continuous inverse on $X^{*}$, and let $\Psi: X \rightarrow \mathbb{R}$ be a sequentially weakly lower semicontinuous, continuously Gâteaux differentiable functional whose Gâteaux derivative is compact such that

$$
\Phi(0)=\Psi(0)=0 .
$$

Assume that there exist $r>0$ and $\bar{x} \in X$, with $r<\Phi(\bar{x})$ such that

(i) $\sup _{\Phi(x) \leq r} \Psi(x)<r \Psi(\bar{x}) / \Phi(\bar{x})$,

(ii) for each $\lambda$ in

$$
\Lambda_{r}:=\left(\frac{\Phi(\bar{x})}{\Psi(\bar{x})}, \frac{r}{\sup _{\Phi(x) \leq r} \Psi(x)}\right),
$$

the functional $\Phi+\lambda \Psi$ is coercive.

Then, for each $\lambda \in \Lambda_{r}$, the functional $\Phi+\lambda \Psi$ has at least three distinct critical points in $X$. 


\section{MAin RESUlts}

In this section, we present our main results.

Let $\tau:=\sup _{x \in \Omega} \operatorname{dist}(x, \partial \Omega)$. Simple calculations show that there is $x^{0} \in \Omega$ such that $B\left(x^{0}, \tau\right) \subset$ $\Omega$, where $B\left(x^{0}, \tau\right)$ denotes the ball in $\mathbb{R}^{N}$ with center $x^{0}$ and radius of $\tau$. Also, put

$$
\sigma_{p, N}(\tau):=\int_{\frac{\tau}{2}}^{\tau}\left|\tau^{3}(N+1)-8 \tau^{2} s(N+2)+15 \tau s^{2}(N+3)-8 s^{3}(N+4)\right|^{p} s^{N-1} d s
$$

Finally, let us denote

$$
K_{p, N}(\tau):=\frac{\tau^{6 p} \Gamma\left(\frac{N}{2}\right)}{2^{6 p+1}(3 k)^{p} \pi^{\frac{N}{2}} \sigma_{p, N}(\tau)},
$$

where $\Gamma$ denotes the Gamma function and $k$ is defined by (2.2).

Theorem 3.1. Let $f \in C^{0}(\bar{\Omega} \times \mathbb{R})$ and denote $F(x, \xi):=\int_{0}^{\xi} f(x, t) d t$ for all $(x, \xi) \in \bar{\Omega} \times \mathbb{R}$. Assume that there exist two positive constants $c$ and $d$, with $d>c\left(K_{p, N}(\tau)\right)^{\frac{1}{p}}$ such that

$\left(f_{1}\right) F(x, \xi) \geq 0$ for each $(x, \xi) \in\left(\Omega \backslash B\left(x^{0}, \frac{\tau}{2}\right)\right) \times[0, d]$;

$\left(f_{2}\right) \operatorname{meas}(\Omega) \sup _{(x, \xi) \in \Omega \times[-c, c]} F(x, \xi) \leq\left(\frac{c}{d}\right)^{p} K_{p, N}(\tau) \int_{B\left(x^{0}, \frac{\tau}{2}\right)} F(x, d) d x$, where meas $(\Omega)$ is the Lebesgue measure of $\Omega$;

$\left(f_{3}\right)$ there exist a function $\alpha \in L^{1}(\Omega)$ and a positive constant $\gamma<p$ such that

$$
F(x, \xi) \leq \alpha(x)\left(1+|\xi|^{\gamma}\right)
$$

for almost every $x \in \Omega$ and for every $\xi \in \mathbb{R}$.

Then, there exist a number $\rho \in \mathbb{R}$ and an open interval $\Lambda \subseteq[0,+\infty)$ with the following property: for every $\lambda \in \Lambda$ and for every $L^{1}$-Carathéodory function $g: \Omega \times \mathbb{R} \rightarrow \mathbb{R}$ with

$\left(g_{1}\right) \sup _{\{|s| \leq \xi\}}|g(\cdot, s)| \in L^{1}(\Omega)$, for all $\xi>0$,

there exists $\delta>0$ such that, for each $\mu \in[0, \delta]$, problem (1.1) has at least three weak solutions whose norms are less than $\rho$.

Proof. Our aim is to apply Theorem 2.2 with $X=W^{3, p}(\Omega) \cap W_{0}^{1, p}(\Omega)$ endowed with the norm introduced in (2.1). We take $\Phi, \Psi, J$ as in the previous section. Put

$$
I_{\lambda, \mu}(u):=\Phi(u)+\lambda \Psi(u)+\mu J(u)
$$

for $u \in X$ and $\lambda, \mu \in[0,+\infty)$. Note that the weak solutions of (1.1) are exactly the critical points of $I_{\lambda, \mu}$.

If we fix $u \in X$ with $\|u\|>1$, by condition $\left(f_{3}\right)$ and for each $\lambda>0$, we have

$$
\begin{aligned}
\Phi(u)+\lambda \Psi(u) & \geq \frac{\|u\|^{p}}{p}-\lambda \int_{\Omega} \alpha(x)\left(1+|u|^{\gamma}\right) d x \\
& \geq \frac{\|u\|^{p}}{p}-\lambda\|\alpha\|_{L^{1}(\Omega)}\left(1+k^{\gamma}\|u\|^{\gamma}\right) .
\end{aligned}
$$

So, the coercivity of functional $\Phi+\lambda \Psi$ is obtained, and therefore the first assumption of Theorem 2.2 holds true. Next, we put

$$
w(x):= \begin{cases}0 & \text { if } x \in \bar{\Omega} \backslash B\left(x^{0}, \tau\right), \\ 64 \frac{L^{3}}{\tau^{6}}(\tau-L)^{3} d & \text { if } x \in B\left(x^{0}, \tau\right) \backslash B\left(x^{0}, \frac{\tau}{2}\right), \\ d & \text { if } x \in B\left(x^{0}, \frac{\tau}{2}\right)\end{cases}
$$


where

$$
L=\operatorname{dist}\left(x, x^{0}\right)=\sqrt{\sum_{i=1}^{N}\left(x_{i}-x_{i}^{0}\right)^{2}}
$$

Then, we deduce that

$$
\begin{aligned}
& \frac{\partial w(x)}{\partial x_{i}}= \begin{cases}0 & \text { if } \quad x \in \bar{\Omega} \backslash B\left(x^{0}, \tau\right) \cup B\left(x^{0}, \frac{\tau}{2}\right), \\
\frac{64 d\left(x_{i}-x_{i}^{0}\right)}{\tau^{6}}\left(3 L \tau^{3}-12 \tau^{2} L^{2}+15 \tau L^{3}-6 L^{4}\right) & \text { if } \quad x \in B\left(x^{0}, \tau\right) \backslash B\left(x^{0}, \frac{\tau}{2}\right),\end{cases} \\
& \frac{\partial^{2} w(x)}{\partial x_{i}^{2}}=\left\{\begin{array}{lr}
0 & \text { if } x \in \bar{\Omega} \backslash B\left(x^{0}, \tau\right) \cup B\left(x^{0}, \frac{\tau}{2}\right), \\
\frac{64 d}{\tau^{6}}\left(3 L \tau^{3}-12 \tau^{2} L^{2}+15 \tau L^{3}-6 L^{4}\right)+ & \\
\frac{64 d\left(x_{i}-x_{i}^{0}\right)^{2}}{\tau^{6}}\left(\frac{3 \tau^{3}}{L}-24 \tau^{2}+45 \tau L-24 L^{2}\right) & \text { if } x \in B\left(x^{0}, \tau\right) \backslash B\left(x^{0}, \frac{\tau}{2}\right),
\end{array}\right. \\
& \sum_{i=1}^{N} \frac{\partial^{2} w(x)}{\partial x_{i}^{2}}=\left\{\begin{array}{lr}
0 & \text { if } x \in \bar{\Omega} \backslash B\left(x^{0}, \tau\right) \cup B\left(x^{0}, \frac{\tau}{2}\right), \\
\frac{64 d}{\tau^{6}}\left(3 L \tau^{3}(N+1)-12 \tau^{2} L^{2}(N+2)+\right. & \text { if } x \in B\left(x^{0}, \tau\right) \backslash B\left(x^{0}, \frac{\tau}{2}\right),
\end{array}\right. \\
& \frac{\partial \Delta w(x)}{\partial x_{i}}= \begin{cases}0 & \text { if } x \in \bar{\Omega} \backslash B\left(x^{0}, \tau\right) \cup B\left(x^{0}, \frac{\tau}{2}\right), \\
\frac{64 d\left(x_{i}-x_{i}^{0}\right)}{\tau^{6}}\left(\frac{3 \tau^{3}(N+1)}{L}-24 \tau^{2}(N+2)+45 \tau L(N+3)-24 L^{2}(N+4)\right) & \text { if } \quad x \in B\left(x^{0}, \tau\right) \backslash B\left(x^{0}, \frac{\tau}{2}\right),\end{cases}
\end{aligned}
$$

and

$$
|\nabla \Delta w(x)|=\frac{64 d}{\tau^{6}}\left|3 \tau^{3}(N+1)-24 \tau^{2} L(N+2)+45 \tau L^{2}(N+3)-24 L^{3}(N+4)\right| .
$$

Now, if we let $r=\frac{1}{p}\left(\frac{c}{k}\right)^{p}$, then

$$
\Phi(w)=\frac{\|w\|^{p}}{p}=\frac{2^{6 p+1}(3 d)^{p} \pi^{\frac{N}{2}}}{p \tau^{6 p} \Gamma\left(\frac{N}{2}\right)} \sigma_{p, N}(\tau)>\frac{d^{p}}{p k^{p} K_{p, N}(\tau)}>\frac{1}{p}\left(\frac{c}{k}\right)^{p}=r .
$$

Since, $0 \leq w(x) \leq d$, for each $x \in \Omega$, the condition $\left(f_{1}\right)$ ensures that

$$
\int_{\Omega \backslash B\left(x^{0}, \tau\right)} F(x, w(x)) d x+\int_{B\left(x^{0}, \tau\right) \backslash B\left(x^{0}, \frac{\tau}{2}\right)} F(x, w(x)) d x \geq 0 .
$$

Hence, by condition $\left(f_{2}\right)$ and (3.2), one has

$$
\begin{aligned}
\operatorname{meas}(\Omega) \sup _{(x, \xi) \in \Omega \times[-c, c]} F(x, \xi) & \leq\left(\frac{c}{d}\right)^{p} K_{p, N}(\tau) \int_{B\left(x^{0}, \frac{\tau}{2}\right)} F(x, d) d x \\
& \leq\left(\frac{c}{k\|w\|}\right)^{p} \int_{B\left(x^{0}, \frac{\tau}{2}\right)} F(x, d) d x \\
& \leq\left(\frac{c}{k\|w\|}\right)^{p} \int_{\Omega} F(x, w(x)) d x .
\end{aligned}
$$


Taking $\sup _{x \in \Omega}|u(x)| \leq k\|u\|$ into account, we obtain that $|u(x)| \leq c$ for all $u \in X$ with $\Phi(u) \leq r$. So, by (3.2) and (3.3), one has

$$
\begin{aligned}
\sup _{\Phi(u) \leq r}(-\Psi(u)) & \leq \int_{\Omega} \sup _{\Phi(u) \leq r} F(x, u) d x \\
& \leq \int_{\Omega|t| \leq c} \sup _{|t| \leq} F(x, u) d x \\
& \leq \operatorname{meas}(\Omega) \sup _{(x, \xi) \in \Omega \times[-c, c]} F(x, \xi) \\
& \leq\left(\frac{c}{k\|w\|}\right)^{p} \int_{\Omega} F(x, w(x)) d x=r \frac{-\Psi(w)}{\Phi(w)} .
\end{aligned}
$$

Fixing any $v>1$, it is easy to see that

$$
\sup _{u \in \Phi^{-1}(]-\infty, r[)}(-\Psi(u))+\frac{r \frac{-\Psi(w)}{\Phi(w)}-\sup _{u \in \Phi^{-1}(]-\infty, r[)}(-\Psi(u))}{v}<r \frac{-\Psi(w)}{\Phi(w)} .
$$

If $h$ satisfies

$$
\sup _{u \in \Phi^{-1}(]-\infty, r[)}(-\Psi(u))+\frac{r \frac{-\Psi(w)}{\Phi(w)}-\sup _{u \in \Phi^{-1}(]-\infty, r[)}(-\Psi(u))}{v}<h<r \frac{-\Psi(w)}{\Phi(w)},
$$

applying Theorem 2.3 with $u_{0}=0$ and $u_{1}=w$, we obtain

$$
\sup _{\lambda \geq 0} \inf _{x \in X}(\Phi(x)+\lambda(\Psi(x)+h))<\inf _{x \in X} \sup _{\lambda \geq 0}(\Phi(x)+\lambda(\Psi(x)+h)) .
$$

Therefore, the assumption (2.3) of Theorem 2.2 holds true.

Let $h_{1} \in C(\Omega)$ be a positive function and $h_{2} \in C(\mathbb{R})$ be a function. Let $f(x, u)=h_{1}(x) h_{2}(u)$ for each $(x, u) \in \Omega \times \mathbb{R}$,

$$
H(t)=\int_{0}^{t} h_{2}(\xi) d \xi
$$

for all $t \in \mathbb{R}$ and $\alpha_{1}(x)=\frac{\alpha(x)}{h_{1}(x)}$. Then, applying Theorem 3.1, we have following result.

Theorem 3.2. Assume that there exist two positive constants $c$ and $d$, with $d>c\left(K_{p, N}(\tau)\right)^{\frac{1}{p}}$ such that

$\left(f_{1}\right) F(x, \xi) \geq 0$ for each $(x, \xi) \in\left(\Omega \backslash B\left(x^{0}, \frac{\tau}{2}\right)\right) \times[0, d]$;

$\left(f_{2}\right) \operatorname{meas}(\Omega) \sup _{(x, \xi) \in \Omega \times[-c, c]} F(x, \xi) \leq\left(\frac{c}{d}\right)^{p} K_{p, N}(\tau) \frac{H(d)}{H(c)} \int_{B\left(x^{0}, \frac{\tau}{2}\right)} h_{1}(x) d x$;

$\left(f_{3}\right)$ there exist a negative function $\alpha \in L^{1}(\Omega)$ and a positive constant $\gamma<p$ such that

$$
F(x, \xi) \leq \alpha_{1}(x)\left(1+|\xi|^{\gamma}\right),
$$

for almost every $x \in \Omega$ and for every $\xi \in \mathbb{R}$.

Then, there exist a real positive $\rho \in \mathbb{R}$ and an open interval $\Lambda \subseteq[0,+\infty)$ with the following property: for every $\lambda \in \Lambda$ and for every $L^{1}$-Carathéodory function $g: \Omega \times \mathbb{R} \rightarrow \mathbb{R}$, thus satisfying

$\left(g_{1}\right) \sup _{\{|s| \leq \xi\}}|g(\cdot, s)| \in L^{1}(\Omega)$, for all $\xi>0$, 
there exists $\delta>0$ such that, for each $\mu \in[0, \delta]$, the problem

$$
\left\{\begin{array}{l}
-\Delta_{p}^{3} u=\lambda h_{1}(x) h_{2}(u)+\mu g(x, u), \quad x \in \Omega, \\
u=\Delta u=\Delta^{2} u=0, \quad x \in \partial \Omega,
\end{array}\right.
$$

has at least three weak solutions whose norms are less than $\rho$.

A simple consequence of Theorem 3.2 is as follows.

Theorem 3.3. Let $f: \mathbb{R} \rightarrow \mathbb{R}$ be a continuous function. Put $F(\xi)=\int_{\Omega} f(t) d t$ for $\xi \in \mathbb{R}$ and assume that there exist two positive constants $c$ and $d$, with $d>c\left(K_{p, N}(\tau)\right)^{\frac{1}{p}}$ such that

$\left(f_{1}\right) F(\xi) \geq 0$ for each $\xi \in[0, d]$;

$\left(f_{2}\right) \operatorname{meas}(\Omega) \sup _{[-c, c]} F(\xi) \leq\left(\frac{c}{d}\right)^{p} K_{p, N}(\tau) \frac{\tau^{N} \pi^{\frac{N}{2}}}{2^{N-1} \Gamma\left(\frac{N}{2}\right)} F(d)$;

$\left(f_{3}\right)$ there exist a negative constant $\alpha$ and a positive constant $\gamma<p$ such that

$$
F(\xi) \leq \alpha\left(1+|\xi|^{\gamma}\right)
$$

for every $\xi \in \mathbb{R}$.

Then, there exist a real positive $\rho \in \mathbb{R}$ and an open interval $\Lambda \subseteq[0,+\infty)$ with the following property: for every $\lambda \in \Lambda$ and for every $L^{1}$-Carathéodory function $g: \Omega \times \mathbb{R} \rightarrow \mathbb{R}$, thus satisfying

$\left(g_{1}\right) \sup _{\{|s| \leq \xi\}}|g(\cdot, s)| \in L^{1}(\Omega)$, for all $\xi>0$,

there exists $\delta>0$ such that, for each $\mu \in[0, \delta]$, the problem

$$
\left\{\begin{array}{l}
-\Delta_{p}^{3} u=\lambda f(u)+\mu g(x, u), \quad x \in \Omega, \\
u=\Delta u=\Delta^{2} u=0, \quad x \in \partial \Omega,
\end{array}\right.
$$

has at least three weak solutions whose norms are less than $\rho$.

Our other main result reads as follows.

Theorem 3.4. Let $f \in C^{0}(\bar{\Omega} \times \mathbb{R})$ and denote $F(x, \xi):=\int_{0}^{\xi} f(x, t) d t$ for all $(x, \xi) \in \bar{\Omega} \times \mathbb{R}$. Assume that there exist two positive constants $d$ and $c$, with $d>c\left(K_{p, N}(\tau)\right)^{\frac{1}{p}}$ such that

$\left(f_{1}\right) F(x, \xi) \geq 0$ for each $(x, \xi) \in\left(\Omega \backslash B\left(x^{0}, \frac{\tau}{2}\right)\right) \times[0, d]$;

$$
\frac{\int_{\Omega|t| \leq c} \max _{c^{p}} F(x, \xi) d x}{c^{p}}<K_{p, N}(\tau) \frac{\int_{B}\left(x^{0}, \frac{\tau}{2}\right) F(x, d) d x}{d^{p}} ;
$$

$\left(f_{3}\right)$ there exist a function $\alpha \in L^{1}(\Omega)$ and a positive constant $\gamma<p$ such that

$$
F(x, \xi) \leq \alpha(x)\left(1+|\xi|^{\gamma}\right)
$$

for almost every $x \in \Omega$ and for every $\xi \in \mathbb{R}$.

Then, for every $\lambda$ in

$$
\Lambda:=\left(\frac{2^{6 p+1} 3^{p} \pi^{\frac{N}{2}} \sigma_{p, N}(\tau) d^{p}}{p \tau^{6 p} \Gamma(N / 2) \int_{B\left(x^{0}, \frac{\tau}{2}\right)} F(x, d) d x}, \frac{c^{p}}{p k^{p} \int_{\Omega} \max _{|t| \leq c} F(x, t) d x}\right)
$$

problem

$$
\left\{\begin{array}{l}
-\Delta_{p}^{3} u=\lambda f(x, u), \quad x \in \Omega, \\
u=\Delta u=\Delta^{2} u=0, \quad x \in \partial \Omega
\end{array}\right.
$$


has at least three weak solutions.

Proof. Let $X, \Phi, \Psi$ and $w$ be as in the proof of Theorem 3.1. Using Theorem 2.4, we obtain the desired conclusion immediately.

\section{Acknowledgements}

This research was supported by a grant from Gonbad Kavous University (No. 6/354).

\section{REFERENCES}

[1] G.A. Afrouzi and S. Shokooh, Existence of infinitely many solutions for quasilinear problems with a $p(x)$ biharmonic operator, Electron. J. Differ. Equ. 317 (2015), 1-14.

[2] F. Cammaroto and L. Vilasi, Multiple solutions for a Kirchhoff-type problem involving the $p(x)$ - biharmonic operator, Nonlinear Anal. 74 (2011), 1841-1852.

[3] B. Ge, Q.M. Zhou, Y.H. Wu, Eigenvalues of the $p(x)$-biharmonic operator with indefinite weight, Zeitschrift für angewandte Mathematik und Physik, 66 (2015), 1007-1021.

[4] L. Kong, Multiple solutions for fourth order elliptic problems with $p(x)$-biharmonic operators, Opusc. Math. 36 (2016), 253-264.

[5] E. Acerbi, G. Mingione, Regularity results for a class of functionals with non-standard growth, Arch. Ration. Mech. Anal. 156 (2001), 121-140.

[6] M. Růžička, Electrorheological Fluids: Modeling and Mathematical Theory, vol. 1748 of Lecture Notes in Mathematics, Springer, Berlin, Germany, 2000.

[7] W.M. Winslow, Induced fibration of suspensions, J. Appl. Phys. 20 (1949), 1137-1140.

[8] B. Rahal, Existence results of infinitely many solutions for $p(x)$-Kirchhoff type triharmonic operator with Navier boundary conditions, J. Math. Anal. Appl. 478 (2019), 1133-1146.

[9] P. Candito, G. Molica Bisci, Multiple solutions for a Navier boundary value problem involving the $p$ - biharmonic operator, Discrete Contin. Dyn. Syst. Ser. S. 5 (2012), 741-751.

[10] P. Candito, L. Li, R. Livrea, Infinitely many solutions for a perturbed nonlinear Navier boundary value problem involving the $p$-biharmonic, Nonlinear Anal. 75 (2012), 6360-6369.

[11] S. Shokooh, G.A. Afrouzi, Existence results of infinitely many solutions for a class of $p(x)$ - biharmonic problems, Comput. Methods Differ. Equ. 5 (2017), 310-323.

[12] H. Yin, Y. Liu, Existence of three solutions for a Navier boundary value problem involving the $p(x)$ biharmonic, Bull. Korean. Math. Soc. 6 (2013), 1817-1826.

[13] H. Yin, M. Xu, Existence of three solutions for a Navier boundary value problem involving the $p(x)$ biharmonic operator, Ann. Polon. Math. 109 (2013), 47-54.

[14] B. Ricceri, A three critical points theorem revisited, Nonlinear Anal. 9 (2009), 3084-3089.

[15] P. Drábek, J. Milota, Methods of nonlinear analysis: Application to differential equations, Birkhäuser, Berlin, 2006.

[16] X.L. Fan, D. Zhao, On the spaces $L^{p(x)}(\Omega)$ and $W^{m, p(x)}(\Omega)$, J. Math. Anal. Appl. 263 (2001), 424-446.

[17] G. A. Afrouzi, V. Rădulescu, S. Shokooh, Multiple solutions of Neumann problems: an Orlicz-Sobolev Space Setting, Bull. Malays. Math. Sci. Soc. 40 (2017), 1591-1611.

[18] G. Bonanno, Some remarks on a three critical points theorem, Nonlinear Anal. 54 (2003), 651-665.

[19] G. Bonanno, S.A. Marano, On the structure of the critical set of non-differentiable functionals with a weak compactness condition, Appl. Anal. 89 (2010), 1-10. 